\title{
Prevalencia de enteroparásitos en niños de la etnia Yukpa de Toromo, Estado Zulia, Venezuela
}

\author{
Iris Díaz $A^{1 a}$, Zulbey Rivero $R^{2 a}$, Ángela Bracho $M^{a}$, \\ María Castellanos $\mathrm{S}^{\mathrm{a}}$, Ellen Acurerola, \\ Marinella Calchi $L^{1 a}$, Ricardo Atencio $\mathrm{T}^{3 \mathrm{a}}$. \\ Prevalence of Intestinal Parasites in \\ Children of Yukpa Ethnia in Toromo, \\ Zulia State, Venezuela
}

Background: Yupkpa community in Perijá Sierra does not have drinking water or sewage elimination systems. Thus it becomes a propitious place for infection with intestinal parasites. Aim: To determine the prevalence of intestinal parasites in children, ages 0 to 14 years, in the Yukpa ethnic population of Toromo in the Perijá Mountains, Zulia State, Venezuela. Material and Methods: Ninety one fecal samples were analyzed by the following coproparasitologic methods: saline solution and lugol fresh mount, formalin-ether concentration (Ritchie), and Kinyoun stain for intestinal coccidians. Results: Overall parasite infection prevalence was $83.5 \%$. The most frequently observed parasites were: Ascaris lumbricoides $(57.1 \%)$, Trichuris trichiura (20.8\%), Hymenolepis nana (14.2\%), Blastocystis hominis (51.6\%), Giardia lamblia (30.7\%), and Entamoeba histolytica/E. dispar complex (21.9\%). Coccidians were nont observed in any of the stool samples. Conclusions: The large number of infected people is directly related to the hygienic and sanitary conditions of the population studied (Rev Méd Chile 2006; 134: 72-8).

(Key words: Indians, South American; Parasites; Parasitology; Venezuela)

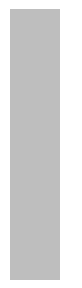

\footnotetext{
Recibido el 8 de julio, 2004. Aceptado el 17 de diciembre, 2005.

El trabajo se autofinanció con materiales de las cátedras de Parasitología y P. P. de Parasitología de la Universidad del Zulia, por tanto fue diseñado, preparado y revisado por los docentes de estas cátedras que participaron en el estudio.

${ }^{1}$ Cátedra de Parasitología, Escuela de Bioanálisis, La Universidad del Zulia.

${ }^{2}$ Cátedra Práctica Profesional de Parasitología, Escuela de Bioanálisis, La Universidad del Zulia. 3Laboratorio Regional de Referencia Virológica, Escuela de Bioanálisis, La Universidad del Zulia. aLicenciado en Bioanálisis.
}

\footnotetext{
Correspondencia a: Prof. Zulbey Rivero Reyes de Rodríguez. Venezuela, Estado Zulia, Maracaibo. Urb. La Rotaria Av 87 \#82-36. Telf: 0058-0261-7541650. Escuela de Bioanálisis, Facultad de Medicina. Universidad del Zulia. Final Av. 20 Telf: 0058-0261-7597219. Fax: 0058-0261-7597224.

E-mail: zulbeyrivero@cantv.net
} 
L as parasitosis intestinales constituyen un grupo de enfermedades con una alta prevalencia, las cuales comprometen al individuo, a la familia y a la comunidad. Desde el punto de vista epidemiológico, la contaminación fecal del suelo, el deficiente saneamiento ambiental y la mala higiene personal son, sin duda, los factores más determinantes que condicionan estas enfermedades; es decir, no sólo las carencias económicas, sino las culturales, afectan al individuo en su salud.

Se ha demostrado que los programas de atención primaria de salud bien aplicados son efectivos en la lucha contra las parasitosis intestinales; por lo que se hace necesario una implementación de estos programas de salud enfocados en la educación sanitaria y el saneamiento ambiental ${ }^{1}$.

Las poblaciones indígenas se encuentran dentro de los grupos más vulnerables a las parasitosis, debido a que tienen ingresos bajos, viven en condiciones deficientes y carecen de acceso adecuado al empleo, educación, el agua potable, la alimentación y los servicios de atención de salud.

La comunidad Yukpa de la Sierra de Perijá no cuenta con red de aguas, ni lugares adecuados para el depósito de las excretas y basura, lo cual, aunado a los escasos recursos económicos, dan lugar propicio para el desarrollo de diversas enfermedades, en especial las enteroparasitosis; generalmente, las viviendas están constituidas por un solo ambiente, albergando a un elevado número de habitantes, lo que conlleva a que se instale el hacinamiento.

Estudios realizados en 1992 en la comunidad indígena de Santa Ana de Wasama, señalaban que los helmintos de mayor prevalencia fueron Ascaris lumbricoides 36,6\%, seguido de Trichuris trichiura 31,7\%; de igual forma los principales protozoarios eran Entamoeba coli 63,4\% y Entamoeba histolytica 26,8\%. Para el mismo año se investigaron las geohelmintiasis en comunidades indígenas y suburbanas del Estado Zulia, donde se obtuvo una prevalencia de $61,1 \%$ para $\mathrm{T}$ trichiura que ocupó el primer lugar, seguido por Ancylostomideos con $56,0 \%$ y A lumbricoides con $52,3 \% 2,3$.

La inquietud por el presente estudio surgió por la falta de interés y abandono que existe hacia esas comunidades; además, la oportunidad de entrar en contacto con esas poblaciones en el medio selvático del Estado Zulia, nos ofrecía la posibilidad de estudiar las enteroparasitosis, y los factores socioeconómicos que condicionan la prevalencia de la misma en esas zonas.

\section{MATERIALES Y MÉTODOS}

Descripción de la población. El estudio fue realizado en una comunidad indígena de la Sierra de Perijá, Municipio Machiques de Perijá, ubicada al Oeste del Estado Zulia ${ }^{4}$.

Partiendo de la clasificación de las etnias indígenas en Venezuela y tomando como punto de partida las familias lingüísticas, los yukpas, pertenecen a los Caribes, cuya organización social está caracterizada por la formación de comunidades relativamente pequeñas. Generalmente, su alimentación se basa en frutas silvestres; trabajan la cesterpía y son monógamos ${ }^{5}$.

La actividad económica más difundida es la agricultura, siendo su principal cultivo el café, el cual comercializan en el Fondo Nacional del Café y en el Mercado de Machiques; también cultivan aguacate, yuca, ñame, ocumo, plátano, cambur, maíz, etc.; de igual forma desarmollan la ganadería 6 .

Esta población, se caracteriza por sus precarias condiciones sanitarias, donde hay ausencia de sistemas para disposición de excretas y no hay agua potable; el agua de consumo la obtienen directamente del río y la almacenan en recipientes sin tapa. En el poblado existen una escuela y una iglesia, así mismo poseen un ambulatorio en el cual se presta atención médica cada 15 días.

Metodología de Laboratorio. Se seleccionaron al azar 91 niños de ambos sexos con edades comprendidas entre 0 y 14 años, distribuidos de la siguiente forma: 1 de 0 a 28 días; 13 de 1 a 11 meses; 5 de 12 a 23 meses; 39 de 2 a 6 años y 33 de 7 a 14 años; se recolectó una muestra fecal por individuo. A los adultos representantes se les solicitó su consentimiento para la realización de los exámenes y se les proporcionaron los envases recolectores necesarios; además se les dio una explicación verbal sobre la correcta recolección de la muestra.

Las muestras fueron examinadas macroscópica y microscópicamente a través del examen directo con solución salina fisiológica y $\operatorname{lugol}^{7}$, de igual 
forma se realizó un frotis, el cual fue fijado con metanol para posterior coloración; el resto de la muestra fue preservada con formol salino al 7\%, material que fue llevado al Laboratorio de Parasitología de la Escuela de Bioanálisis de la Facultad de Medicina de la Universidad del Zulia. A las muestras preservadas se les practicó el método de concentración de Ritchie (Formol-Eter) ${ }^{7}$ y el frotis fue utilizado para realizar la coloración de Kinyoun $^{8}$, útil para la investigación de coccidios intestinales.

Metodología estadística. Se efectuaron tablas y gráficos de los datos obtenidos en los resultados para representar las principales variables en estudio, tales como parasitosis y sexo, parasitosis y edad, monoparasitismo y poliparasitismo, entre otras. Las edades fueron estratificadas según Quintero ${ }^{9}$. Se empleó el paquete estadístico SAS (Statistical Analysis System) para Windows versión 6.12. (SAS Institute Inc. Campus Drive, Cary, North Caroline, USA).

\section{RESULTADOS}

Del total de las muestras estudiadas (91), se encontró una prevalencia de enteroparasitosis de $83,52 \%$ representado por 76 individuos parasitados.

En relación a las variables parasitismo y sexo, se obtuvieron los siguientes resultados: el mayor porcentaje de parasitados se observó en el sexo masculino con 85,71\%; mientras que el sexo femenino, representó el 82,14\% (Figura 1).
En cuanto a la distribución por grupo etario, se obtuvieron los siguientes resultados; dentro del grupo de parasitados, en el grupo de 7 a 14 años la totalidad de los niños estudiados presentó parasitosis (100\%), seguido del grupo de edades entre 2 y 6 años con 92,30\% y del de 12 a 23 meses con un porcentaje de 60,00\%. (Tabla 1 ).

En la Tabla 2, se muestran las especies parasitarias identificadas en el estudio, donde A lumbricoides fue el helminto de mayor prevalencia (52 casos) alcanzando 57,14\%, seguido por T trichiura (19 casos) con 20,88\%; entre los protozoarios B hominis (47 casos) con $51,65 \%$ y Endolimax nana (34 casos) con 37,36\% fueron los más prevalentes. Sin embargo, cabe destacar que a pesar de no ocupar los primeros lugares, los microorganismos potencialmente patógenos G lamblia y el complejo E histolytica/E dispar con 20 casos $(30,77 \%)$ y 28 casos $(21,98 \%)$ respectivamente presentaron un porcentaje bastante relevante. Es importante señalar que aunque se emplearon métodos de coloración específicos para coccidios intestinales no se detectó la presencia de Cryptosporidium sp, Cyclospora cayetanensis o Isospora belli.

En cuanto al tipo de parasitismo encontrado en la población estudiada, se observó el predominio del poliparasitismo $(84,21 \%)$ sobre el monoparasitismo $(15,79 \%)$.

$\mathrm{Al}$ analizar las variables poliparasitismo y monoparasitismo en relación con el grupo etario y el sexo, en el grupo femenino de 2 a 6 años se encontró el mayor porcentaje de poliparasitismo con 27,63 (Tabla 3).

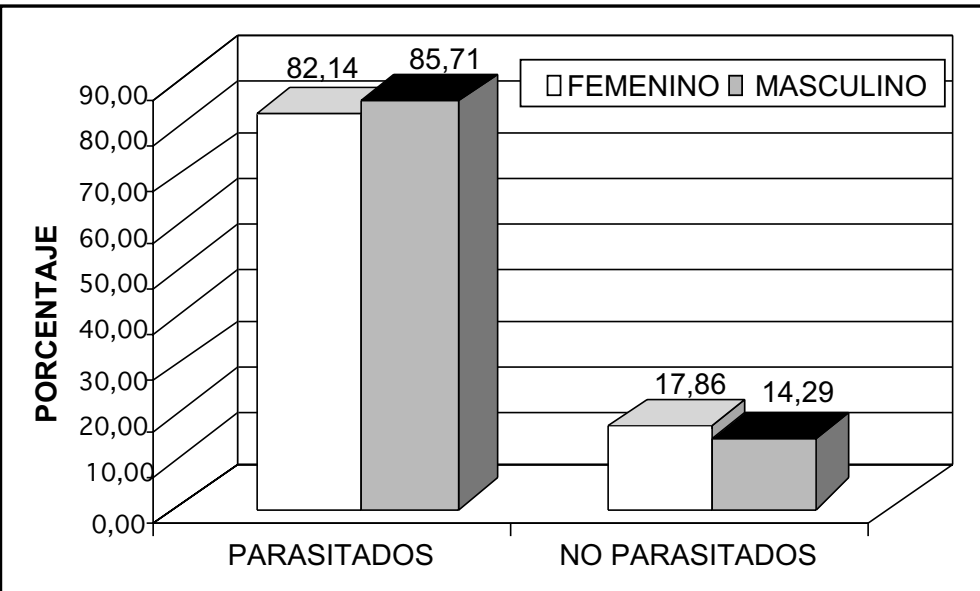

Figura 1. Prevalencia de parasitismo por sexo en niños de la etnia Yukpa, población de Toromo, Estado Zulia. Venezuela, 2002 
Tabla 1. Prevalencia de enteroparasitos por grupo etario en N iños de la etnia Yukpa, población de Toromo, Estado Zulia. Venezuela, 2002

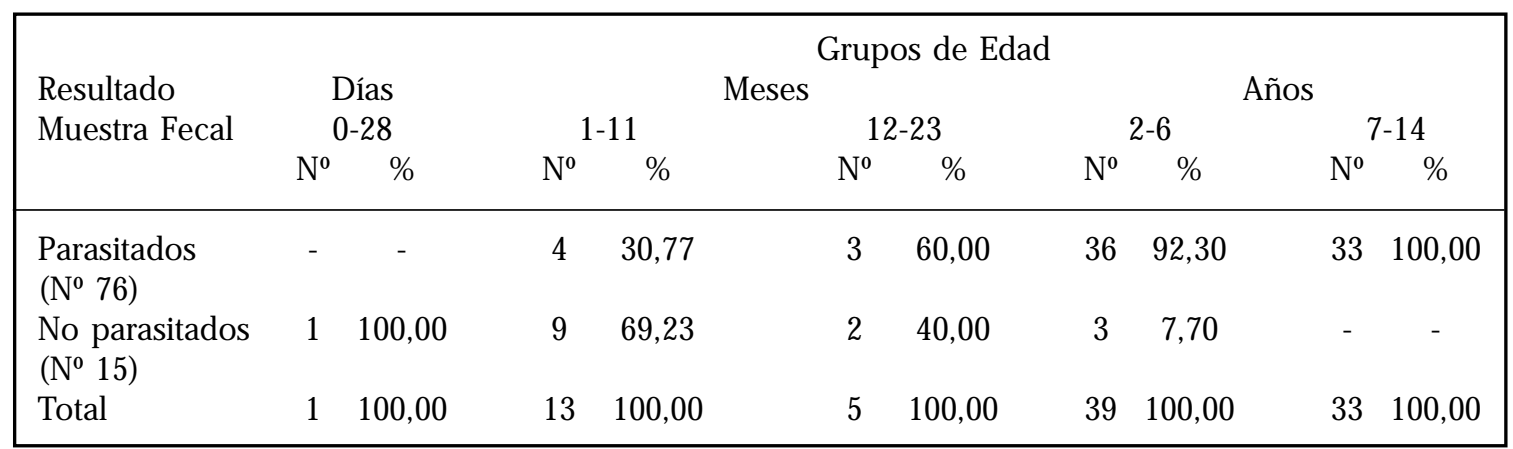

Tabla 2. Prevalencia de especies parasitarias en niños de la etnia Yukpa, población de Toromo, Estado Z ulia. Venezuela, 2002.

\begin{tabular}{|c|c|c|}
\hline Especies Parasitarias & $\begin{array}{l}\text { № de } \\
\text { Casos }\end{array}$ & Prevalencia \\
\hline \multicolumn{3}{|l|}{ Helmintos } \\
\hline Ascaris Lumbricoides & 52 & $57,14 \%$ \\
\hline Trichuris trichiura & 19 & $20,88 \%$ \\
\hline Hymenolepis nana & 13 & $14,29 \%$ \\
\hline Ancylostomideos & 4 & $4,40 \%$ \\
\hline Strongyloides stercoralis & 2 & $2,20 \%$ \\
\hline \multicolumn{3}{|l|}{ Protozoarios } \\
\hline Blastocystis hominis & 47 & $51,65 \%$ \\
\hline Endolimax nana & 34 & $37,36 \%$ \\
\hline Entamoeba coli & 33 & $36,26 \%$ \\
\hline Giardia lamblia & 28 & $30,77 \%$ \\
\hline Complejo Entamoeba histolytica/E dispar & 20 & $21,98 \%$ \\
\hline Iodamoeba butschlii & 16 & $17,58 \%$ \\
\hline Pentatrichomonas hominis & 12 & $13,19 \%$ \\
\hline Chilomastix mesnili & 4 & $4,40 \%$ \\
\hline
\end{tabular}

Tabla 3. Poliparasitismo y monoparasitismo según grupo etario y sexo en niños de la etnia Yukpa, población de Toromo, estado Zulia. Venezuela, 2002

\begin{tabular}{|c|c|c|c|c|c|c|c|c|}
\hline \multirow[t]{4}{*}{ Grupo Etario } & \multicolumn{8}{|c|}{ Sexo } \\
\hline & \multicolumn{4}{|c|}{ Femenino } & \multicolumn{4}{|c|}{ Masculino } \\
\hline & \multicolumn{2}{|c|}{ Monoparasitosis } & \multicolumn{2}{|c|}{ Poliparasitosis } & \multicolumn{2}{|c|}{ Monoparasitosis } & \multicolumn{2}{|c|}{ Poliparasitosis } \\
\hline & $\mathrm{n}$ & $\%$ & $\mathrm{n}$ & $\%$ & $\mathrm{n}$ & $\%$ & $\mathrm{n}$ & $\%$ \\
\hline $0-11$ meses & 0 & 0,00 & 1 & 1,32 & 3 & 3,95 & 0 & 0,00 \\
\hline $12-23$ meses & 0 & 0,00 & 1 & 1,32 & 1 & 1,32 & 1 & 1,32 \\
\hline 2 a 6 años & 2 & 2,63 & 21 & 27,63 & 4 & 5,26 & 9 & 11,84 \\
\hline 7 a 14 años & 2 & 2,63 & 19 & 25,00 & 0 & 0,00 & 12 & 15,79 \\
\hline Total & 4 & 5,26 & 42 & 55,26 & 8 & 10,53 & 22 & 28,95 \\
\hline
\end{tabular}




\section{DisCUSIÓN}

La estadística real sobre la prevalencia de las parasitosis intestinales puede obtenerse mediante la realización de exámenes coproparasitológicos, ya que la mayoría de estas entidades cursan de manera asintomática ${ }^{10}$.

En el estudio realizado se encontró una alta prevalencia (83,52\%) de enteroparásitos; resultado que al ser comparado con los reportados en otras comunidades indígenas del estado Zulia $^{3}$ se aprecia que son similares. Díaz y $\operatorname{cols}^{3}$ al estudiar las comunidades de Santa Ana de Wasama y Kasmera obtuvieron resultados de: 90,2\% y 93,1\% respectivamente, esta investigación fue realizada en individuos del grupo étnico Yukpa y se puede observar que la prevalencia se mantiene, este hecho debe causar preocupación, porque las cifras indican que con el paso de los años en lugar de disminuir existe un aumento progresivo de la prevalencia de enteroparasitosis en esta etnia. Estas similitudes en los resultados probablemente se deban a que la situación sanitaria en estas regiones cambia muy poco o casi nada, pues la ausencia de sistemas para la eliminación de excretas, la falta de suministro de agua potable, la inexistencia de servicios asistenciales, la malnutrición y la falta de educación sanitaria, son características comunes en el modo de vida de estas comunidades ${ }^{3}$.

Con relación a la prevalencia de parasitosis por sexo, no se encontró diferencia significativa, resultado que concuerda con los obtenidos por Díaz y cols ${ }^{11}$ en diferentes poblaciones indígenas; de igual forma coincide con otras investigaciones realizadas en el país ${ }^{12-14}$.

De igual manera que han reseñado otros investigadores ${ }^{15,16}$, se encontró diferencia significativa entre las variables parasitosis y edad, siendo la de mayor prevalencia el grupo etario comprendido entre 7 y 14 años (100\%) seguido por el grupo de 2 a 6 años (93,30\%) resultado que difieren a los obtenidos por otros investigadores $^{12-14}$. Hay que resaltar que para el grupo de 7 a 14 años, todos los individuos presentaron parasitosis, tales hallazgos podrían deberse a la estrecha relación que establecen los niños en edad escolar con las fuentes de infección (suelo contaminado), debido a la práctica de juegos inherentes a estas edades $^{14,15}$.
Al hablar de la prevalencia según especie parasitaria; el helminto que ocupó el primer lugar fue A lumbricoides $(57,14 \%)$ seguido de T trichiura $(20,88 \%)$, resultado que tiene similitud con un estudio realizado en la población de Kasmera ${ }^{3}$. Según Silva ${ }^{17}$ A lumbricoides y $\mathrm{T}$ trichiura, adquieren características especiales de acuerdo a las condiciones ambientales de temperatura y humedad y a la composición físico-química de los suelos. Con frecuencia A lumbricoides y T trichiura se presentan asociados, debido a la similitud de su ciclo biológico externo y a su mecanismo de transmisión, ya que los huevos embrionados de estas especies son muy resistentes, permaneciendo en el suelo por largo tiempo ${ }^{12}$.

El tercer lugar de prevalencia fue ocupado por H nana (14,29\%), resultado similar al encontrado por Rivero y cols ${ }^{12}$; sin embargo, en otros estudios realizados a nivel regional ${ }^{18-20}$, se observó presente este helminto pero en un porcentaje inferior. Según Reyes y col ${ }^{21}$ el hallazgo de esta cestodiasis, se debe a la facilidad con que se transmite dicha parasitosis, bien sea a través de su ciclo monoxénico o heteroxénico y la posible participación de reservorios animales, cuya presencia es abundante en la población de Toromo.

Otros helmintos encontrados fueron: Ancylostomideos $(4,40 \%)$ y Strongyloides stercoralis $(2,20 \%)$ en bajos porcentajes, lo cual según Botero y cols ${ }^{8}$ y Melvin y $\mathrm{col}^{7}$; puede deberse a que no se utilizaron las técnicas ideales para su diagnóstico.

En cuanto a los protozoarios, Blastocystis hominis representado por $51,65 \%$, ocupa el primer lugar, y la cifra guarda relación con otras investigaciones ${ }^{11,14}$. Este es un protozoario intestinal muy controversial en lo que respecta a su patogenicidad, hasta hace poco era descrito como no patógeno, sin embargo, algunas veces se encuentra asociado a múltiples desórdenes intestinales ${ }^{22}$. La patogenicidad puede depender del número de parásitos presentes en las muestras de heces. Vannatta y cols ${ }^{23}$ sugieren que más de cinco microorganismos por campo, con objetivo de 100X, son capaces de producir cierto grado de alteración gastrointestinal y diarrea. En la presente investigación no se realizaron técnicas de recuento de $\mathrm{B}$ hominis ni su relación con cuadros diarreicos.

E nana ocupa el segundo lugar (37,36\%) al igual que lo encontrado por Díaz y cols ${ }^{11}$, sin 
embargo, en la mayoría de los estudios realizados $^{12,14,24}$ ocupa el tercer lugar de prevalencia. En tercer lugar de prevalencia en nuestro estudio, se encuentra E coli (36,26\%). E nana y E coli son comensales del intestino y de acuerdo a su biología, su presencia indica que existe contaminación de los alimentos y del agua ingerida, con residuos fecales ${ }^{12,13}$.

Se pudo observar que G lamblia (30,77\%) y el complejo E histolytica/E dispar (21,98\%) a pesar de ser los patógenos potenciales no ocupan los primeros lugares de parasitismo, resultados que se difieren a los obtenidos por Díaz y cols ${ }^{11}$. Según Botero $^{25}$ y Homez y cols ${ }^{26}$ estos enteroparásitos son responsables de diarreas, dolor abdominal, flatulencia, entre otros, y su presencia confirma que aun cuando existan condiciones sanitarias adecuadas, si no se practica una adecuada higiene personal, estos enteroparásitos persisten, ya que su principal vía de transmisión es oro-fecal, mediante la ingestión de agua y alimentos contaminados con materia fecal donde se encuentran las formas evolutivas infectantes.

La ausencia de coccidios intestinales en las muestras analizadas hace presumir que estamos ante una comunidad que no ha estado expuesta a los ooquistes de estas especies, a pesar de tratarse de individuos que tienen contacto con especies bovinas, uno de los mayores reservorios de ooquistes de Cryptosporidium parvum, ya que para Isospora belli y Cyclospora cayetanensis está transmisión es más relacionada con el género humano, hasta ahora su único hospedero natural ${ }^{8}$.

\section{REFERENCIAS}

1. Boland R, Young M. La estrategia, el costo y el progreso de la atención primaria de salud. Bol Ofic Sanit Panam 1986; 93: 550-62.

2. Chourio G, Morales G, Pino L, Díaz i, araujo M, Rincón W. Geohelmintiasis en comunidades indígenas y suburbanas del estado Zulia. Kasmera 1993; 21: 37-64.

3. Díaz I, Chourio G, Barrios Y, díaz F, Finol R. Enteroparasitosis en comunidades de la etnia Yukpa del Estado Zulia. Kasmera 1994; 22: 1-27.

4. Hinestroza J. Nuestra vida depende de la Sierra de Perijá. (on line) Disponible en: http://csf.colorado.edu/mail/elan/mar97/0058.html
En lo referente al tipo de parasitismo, se encontró un alto índice de poliparasitismo en esta comunidad $^{3}$. Tales hallazgos reflejan las deficiencias sanitarias básicas y socio-culturales que caracterizan a estos grupos.

En la presente investigación, la frecuencia del poliparasitismo aumentó con la edad, lógicamente cuando se establece un mayor contacto con el entorno que obviamente está contaminado con variadas formas evolutivas de parásitos. Esta situación aumenta la posibilidad de adquirir una parasitosis diferente en cada contacto. El grupo femenino de 2-6 años resultó ser el más significativo del total y esto pudiese relacionarse con el hecho de que las niñas de esta edad, no acuden al preescolar y generalmente acompañan a su madre en el área peridomiciliaria, donde la madre se encuentra efectuando las actividades de limpieza, recolección de agua y siembra de vegetales, lo que les permite un amplio contacto con superficies contaminadas.

La importancia de este hecho radica en que se ha observado, por lo menos para las helmintiasis, valores de carga parasitaria más elevada cuando se observan parasitosis asociadas, que como parasitosis únicas. Tomando en cuenta la elevada prevalencia de asociaciones entre helmintos en los niños estudiados (21 casos) y considerando el efecto que estas infecciones parasitarias pueden tener sobre el procesamiento mental y el proceso cognitivo se puede inferir la importancia que tiene el poliparasitismo en los individuos estudiados ${ }^{27-29}$.

5. Información sobre Nuestras etnias indígenas. (on line) Disponible en: http://www.sucreturistico. $\mathrm{com} /$ tipsindigenas.htm

6. Delgado L, Marín H, Aptiz de P A. El Zulia su espacio geográfico. Caracas, Venezuela. 1992. p. 201.

7. Melvin D, BRooke M. Métodos de Laboratorio para el diagnóstico de Parasitosis Intestinales. México. Editorial Interamericano. 1971; 198.

8. Botero D, Restrepo M. Parasitosis Humanas. Segunda Edición. Corporación para investigaciones Biológicas (CIB) Medellín, Colombia. 1992; 3-304.

9. QUINTERO R. Puericultura. Atención primaria en la salud infanto-juvenil. Editorial de la Universidad del Zulia (EDILUZ). 2001. Maracaibo, Venezuela. p: 394. 
10. Díaz I, Flores T. Prevalencia de parásitos intestinales en alumnos de educación básica del Municipio Cacique Mara. Maracaibo, Estado Zulia. Kasmera 1990; 18: 46-70.

11. Díaz A, Botero L, Ledesma F, Molero N, Cotesh M, Jaouhary R et al. Prevalencia de enteroparasitosis en individuos que acuden a la unidad docente asistencial de medicina familiar «uis Sergio Pérez». Kasmera 2000; 28: 45-62.

12. Rivero Z, Chango E, Iriarte H. Enteroparasitosis en alumnos de la escuela básica «r. Jesús María Portillo» del Municipio Maracaibo-Estado Zulia. Kasmera 1997; 25: 121-39.

13. Rvero Z, Chourio G, Díaz I, Cheng R, Rucson G. Enteroparasitosis en escolares de una institución pública del Municipio Maracaibo. Invest Clin 2000; 41: 27-57.

14. Simoes M, Rivero Z, Carreño G, Lugo M, MaidonaDo A, Chacin I et AL. Prevalencia de enteroparasitosis en una escuela urbana en el Municipio San Francisco, Estado Zulia-Venezuela. Kasmera 2000; 28: 27-43.

15. Chourio G, Rincon W, Casteluanos M, Luzardo T, Melean C. Prevalencia Parasitaria en una comunidad suburbana del distrito Maracaibo. Estado Zulia. Kasmera 1988; 16: 30-47.

16. Díaz I, Chourio G, Alvarez M, Añez O, Morón A, Romero E. Prevalencia de Parásitos Intestinales en el barrio Teotiste de Gallegos de la Ciudad de Maracaibo, Estado Zulia. Kasmera 1992; 20: 73-94.

17. Silva R. Alcances ecológicos de la epidemiología de las enfermedades parasitarias. Bol Chil Parasitol 1962-1965; 17-20: 113-22.

18. Calchi M, Chourio G, Díaz I. Helmintiasis Intestinal en niños de una comunidad marginal del Municipio Maracaibo. Estado Zulia-Venezuela. Kasmera 1996; 24: 17-38.

19. Chacin B, Guanipa N, Arape R. Prevalencia de Entamoeba histolytica, Entamoeba hartmani y otras parasitosis intestinales en niños hospitalizados. Invest Clin 1976; 17: 25-41.

20. Rivero Z, Acevedo C, Casanova I, Hernández S, Malsapina A. Enteroparasitosis en escolares de dos unidades educativas rurales del Municipio La Cañada. Estado Zulia-Venezuela. Kasmera 1996; 24: 151-77.

21. Reyes H, Noemí I. Himenolepiasis y Dipilidiasis. En Atias, A. y Neghme, A. Parasitología Clínica. 3a Edición. Publicaciones Técnicas Mediterráneo. Santiago de Chile. 1992; 213-218.

22. Stenzl J, Borehan L Blastocystis hominis. Revisited Clin Microbiol Rev 1996; 9: 563-84.

23. Vannatta $H$, Andanzon D, Mumican K. Blastocystis hominis infection presenting as recurrent diarrhea. Ann Int Med 1985; 102: 495-6.

24. Rivero Z, Díaz I, Acurero E, Camacho M, Medina M, Ríos L. Prevalencia de Parasitosis Intestinales en escolares de 5 a 10 años de un instituto del Municipio Maracaibo. Estado Zulia-Venezuela. Kasmera 2001; 29: 153-70.

25. Botero D. Persistencia de Parasitosis Intestinales Endémicas en América Latina. Bol Ofic Sanit Panam 1981; 90: 39-46.

26. Homez J, Soto R, De Soto S, Méndez R, Mármol P. Parasitología. Editorial de la Universidad del Zulia. 8a Edición. 1995; 23-267.

27. NoKes C, Bundy DAP. Does helminth infection affect mental processing and educational achievement? Parasitology today 1994; 10: 14-18.

28. Simeon D, Cauender J, Wong M, Grantham-McgreGOR S, RAMDATH DD. School perfomance, nutricional status and Trichuriasis in Jamaican schoolchildren. Acta Paediatr 1994; 83: 1188-983.

29. ForRester JE, SCOTT ME, Bundy DAP, GoLdEN MNH. Predisposition of individuals and families in México to infection with A lumbricoides and $\mathrm{T}$ trcichiura. Trans R Soc Trop Med Hyg 1990; 84: 272-6. 